\title{
THE INFLUENCE OF DIFFERENT VENTILATION PATTERNS ON TREATMENT OF PATIENTS WITH SEVERE TRAUMATIC BRAIN INJURY
}

\author{
O. V. Oliynyk ${ }^{1,2}$, B. O. Pereviznyk ${ }^{1}$, A. Shlifirchyk ${ }^{2}$ \\ ${ }^{1}$ I. HORBACHEVSKY TERNOPIL STATE MEDICAL UNIVERSITY, TERNOPIL, UKRAINE \\ ${ }^{2}$ POPE JOHN PAUL II STATE SCHOOL OF HIGHER EDUCATION, BIALA PODLASKA, POLAND
}

Background. Respiratory support is a vital method for temporary compensation of external breathing function in patients with severe traumatic brain injury. However, it is not always possible to deal with severe respiratory dysfunction even with the usage of up-to-date respiratory technologies. This work is aimed to find an answer how different pattern of mechanical ventilation influence on a treatment of patients with severe traumatic brain injury.

Objective. The influence of respiratory support, as a main method for temporary compensation of external breathing function, on treatment result for patients with severe traumatic brain injury.

Methods. Treatment results of 253 patients with severe traumatic brain injury of Ternopil University Hospital were evaluated due to the type of respiratory support used. The results were separately evaluated in alive and dead patients.

Results. Mortality rate of patients depended on the type of mechanical ventilation that was used. The highest mortality (58.69\%) was in the group, when a patient was transferred to forced ventilation a volume control. The mortality rate was decreasing by $51.78 \%$ in case of adding PEEP. The strategy of using accessory lung ventilation patterns CPAP and BiPAP caused significant (in 1.48 times) decrease of mortality in this group of patients.

Conclusions. The survival of patients with severe traumatic brain injury, who were ventilated by the method of consistent combination of forced ventilation with pressure control (CPV) and 2 patterns of accessory lung ventilation: Constant Positive Airway Pressure (CPAP) and Biphasic positive airway pressure (BiPAP), is reliably higher than in the case of forced ventilation with volume control with Positive end-expiratory pressure.

KEY WORDS: severe traumatic brain injury, lung ventilation, treatment, intensive care.

\section{Introduction}

The process of treating the patients with severe traumatic brain injury (STBI) is a topical issue of contemporary medicine [1]. It is established that the mortality rate in case of STBI is $80 \%$, it is caused by the development of multiple organ failure [2].

STBI is one of the causes of mortality and disability all over the world. It is also one of the main reasons of human mortality until the age of 40 [3]. In Great Britain STBI frequency is 1500 people per every 100000 population. 9 patients out of 100000 population die because of traumatic brain injury $[1,2]$. The problem of STBI treatment is today not only medical, but social as well [2-5].

Among the complications of STBI the prominent role is given to lung affection which develops in $70-80 \%$ of patients and becomes

Corresponding author: Oleksandr Oliynyk, Department of Anaesthesiology and Critical Care Medicine, I. Horbachevsky Ternopil State Medical University, 1 Maidan Voli, Ternopil, Ukraine, 46001

Phone number: +380352273341

E-mail: oliynyko@tdmu.edu.ua one of the contributory factors for the worsening of the patient state with the further negative treatment results [4]. Morphological changes of lungs with underlying STBI are detected in $95-97.7 \%$ of cases [4]. The most frequent cause of patients mortality on the $3-5^{\text {th }}$ day of STBI is acute respiratory distress syndrome (ARDS) [2]. It happens in almost every STBI patient with multiple organ failure $[6,7]$.

Respiratory support is a main method for the temporary compensation of external breathing function [8]. However, it is not always possible to deal with severe respiratory dysfunction even using up-to-date respiratory technologies.

The question is: how will it influence the treatment result? This study is aimed to find an answer.

\section{Material and Methods}

Treatment results of 253 patients with STBI of Ternopil University Hospital were evaluated due to the type of respiratory support used. 
The results were separately evaluated in alive and dead patients. As the criteria of treatment effectiveness we used the period of patients stay on mechanical ventilation.

4 different patterns of breathing were used: independent or spontaneous breathing, forced ventilation with volume control (CMV) and forced ventilation with pressure control (CPV). We also used 2 patterns of accessory lung ventilation: Constant Positive Airway Pressure (CPAP) and Biphasic positive airway pressure (BiPAP), a type of ventilation with 2 phases of positive pressure in airway. For ventilation with volume control we used a mechanical ventilation device "Bryz". For ventilation in CPAP, BiPAP and CPV modes we used a mechanical ventilator Carina Drager.

In 2012-2015 years in Ternopil University Hospital there were 253 patients with STBI. Mechanical ventilation was delivered for all these patients. They were divided into 3 groups. In the $1^{\text {st }}$ group patients were ventilated in CMV $(5-6 \mathrm{ml} / \mathrm{kg})$. The strategy in $2^{\text {nd }}$ group was the same except during CMV mechanical ventilation we used positive pressure in the end of expiration (PEEP), which didn't exceed 6-9 mbar. In the $3^{\text {rd }}$ group we used the accessory ventilation. If the saturation of these patients in case of independent breathing decreased more than by $92 \%$, the accessory ventilation through endotracheal tube in CPAP pattern or alternatively tracheostomy was implemented. If saturation was lower than $90 \%$, patients were transferred to BiPAP pattern. In case of full absence of independent breathing, the patient of this group was ventilated in CPV pattern. At this strategy PEEP didn't exceed 6-9 mbar, and inspiratory pressure - 20-22 mbar.

Patients usually died because of multiple organ failure, which was followed by ARDS. ARDS was the most frequent cause of patients' deaths during the first 5 days after the surgery. We used Guidelines for the Management of Severe Traumatic Brain Injury of American Association of Neurosurgeons (2007) as the treatment strategy which was modified according to our conditions [4].

In all groups of patients during spontaneous breathing and mechanical ventilation as well, we tried not only to keep the partial oxygen pressure but also to prevent hypocapnia, so the hyperventilation didn't occur.

Infusion therapy was implemented in normovolemic mode in order to avoid hypotension episodes. Additionally, we tried to keep central venous pressure on the level of $8-10 \mathrm{~mm} \mathrm{Hg}$ and hemodynamic support with the usage of vasopressors was also performed. We also tried to prevent the decrease of average blood pressure (BP) lower than $80 \mathrm{~mm} \mathrm{Hg}$. dopamine and adrenaline were the most frequently used among the vasopressors. The highest point of systolic BP was $160 \mathrm{~mm} \mathrm{Hg}$. As for the infusion therapy we preferred Normal Saline Solution (NSS) or Ringer's solution.

Analgosedation was used for eradicating hyperventilation and synchronization with ventilator. The ventilation was implemented with $\mathrm{PaCO}_{2} 35-40 \mathrm{~mm} \mathrm{Hg}$. PEEP was 5-8 $\mathrm{mm} \mathrm{Hg}$ (not higher than $15 \mathrm{~mm} \mathrm{Hg}$ ). According to the treatment strategy we also cured hyperthermia and anemia, prevented convulsions with carbamazepine and gabantin, used low molecular weight heparins at the absence of hemorrhage. For prevention of stress ulcers was conducted and nutrition therapy was applied. We preferred the fastest administration of enteric nutrition by means of standard food mixes and obligatory glycemia control. Corticosteroids (CS) were used only in case of ARDS development. If intracranial pressure was rising we also used barbiturates. Antibiotic therapy was chosen due to the type of microflora. The drugs of choice were meropenem, colomycin, amikacin.

The mortality rate and ventilation period in each studied group were counted. The ventilation period was counted separately: for those who stayed alive and died.

The permission for research implementation was issued by the Commission on Bioethics of I. Horbachevsky Ternopil State Medical University (protocol №29 from May 20, 2015).

\section{Results}

Mortality rate of patients depended on the type of mechanical ventilation that was used. The highest mortality $(58.69 \%)$ was in the $1^{\text {st }}$ group, when the patient was transferred to CMV pattern in terms of insufficient independent breathing. The mortality rate decreased by $51.78 \%$ when PEEP was added. The strategy of using accessory lung ventilation patterns CPAP and BiPAP caused significant (in 1.48 times) mortality decrease in this group of patients.

The usage of different ventilation patterns caused the changes in the period of patients' stay on mechanical ventilation. In the $1^{\text {st }}$ group (CMV), dead patients were on accessory ventilation for very short period and died quickly. Patients, who stayed alive, adversely, stayed on mechanical ventilation for longer period. The accession of PEEP improved ventilation results: 
dead patients lived longer and those, who stayed alive, were taken of ventilator earlier. The best results were in groups with accessory ventilation patterns CPAP and BiPAP. Mortality in this group was lower than in $1^{\text {st }}$ and $2^{\text {nd }}$ groups. Among the alive patients with such mechanical ventilation support, the process of excluding patients from mechanical ventilation was much easier, though the mortality in this group was in average for a few days longer if compared to $1^{\text {st }}$ and $2^{\text {nd }}$ group.

\section{Discussion}

To our mind, the obtained results depend on peculiarities of ventilation pattern and analgosedation. Anesthesiologists always see that a patient feels pain because vital signs, above all pulse, BP and respiratory rate increase. However, we tried not to use the excessive dosages of analgesics. If there are no signs of pain, we could give no analgesics to a patient at all. In case of even small signs of pain we prescribed narcotic analgesics. Frequently, even not high dosage of analgesics caused the impairment of patients' state. Patient started breathing worse and mechanical ventilation was necessary. The same situation was on in case of hyperventilation, which promoted hypercapnia development. Even small dosages of analgesics caused the decrease in patients' state according to GCS level, partial oxygen pressure in arterial blood less than $60 \mathrm{~mm} \mathrm{Hg}$ and promoted the necessity of mechanical ventilation.

The main differences and advantages of accessory ventilation are: in CPAP pattern the ventilator helps the patient to inhale even if there are 40 inhales per 1 minute. The ventilator will support the positive pressure in the end of exhale on the determined level, which equals 6-9 cm of water. Also, it helps to make every effort of inhale according to determined measurement of inspiratory support (20 mBar). In BiPAP pattern the respiratory rate is maintained by a ventilator. Inhalation startS by the patients' effort to inhale, and then the inspiratory support level is achieved. The important thing is that patient can breathe independently and breathe between respiratory cycles, which are provided by mechanical ventilation. In order to achieve the synchronization with it, we need smaller dosages of analgesics. We observed the smaller interruption of consciousness and breathing due to GCS

During the last year we succeed in mortality decrease almost in 4 times: before the rate was 9 to 100000 and now it is 9 up to 200000 . The main causes of this are 2 main factors: the earlier described strategy of respiratory support and the CS in treatment of ARDS.

The most significant problem of intensive care unit is increase of bacteria resistance to carbapenem. In terms of insufficient financial support it is hard to solve.

Table 1. Treatment results of patients with STBI followed by severe sepsis and ARDS depending on type of respiratory support and CS usage

\begin{tabular}{|l|c|c|c|c|c|}
\hline \multirow{2}{*}{ Ventilation mode } & & $\begin{array}{c}\text { Amount } \\
\text { of patients }\end{array}$ & Average age & $\begin{array}{c}\text { Ventilation period } \\
\text { in days }\end{array}$ & \multirow{2}{*}{ Survival, \% } \\
\hline \multirow{2}{*}{ SV+ IMV } & Alive & 19 & 37.52 & 19.47 & \multirow{2}{*}{58.69} \\
\cline { 2 - 5 } & Dead & 27 & 52.96 & 5.22 & \\
\hline \multirow{2}{*}{ SV+IMV+PEEP } & Alive & 27 & 41.0 & 17.14 & \multirow{2}{*}{51.78} \\
\cline { 2 - 5 } & Dead & 29 & 52.1 & 6.76 & \multirow{2}{*}{39.73} \\
\hline \multirow{2}{*}{ CPAP+BiPAP+CPV } & Alive & 91 & 40.85 & 13.93 & 14.1 \\
\cline { 2 - 5 } & Dead & 60 & 50.0 & & \\
\hline
\end{tabular}

Dead patients

\begin{tabular}{|l|c|c|c|}
\hline & SV+IMV & SV+IMV+PEEP & CPAP+BiPAP+CPV \\
\hline SV+IMV & - & 0.0734 & 0.000050 \\
\hline SV+IMV+PEEP & 0.0734 & - & 0.000099 \\
\hline CPAP+BiPAP+CPV & 0.000050 & 0.000099 & - \\
\hline
\end{tabular}

Alive patients

\begin{tabular}{|l|c|c|c|}
\hline & SV+IMV & SV+IMV+PEEP & CPAP+BiPAP+CPV \\
\hline SV+IMV & - & 0.8194 & 0.1769 \\
\hline SV+IMV+PEEP & 0.8194 & - & 0.4688 \\
\hline CPAP+BiPAP+CPV & 0.1769 & 0.4688 & - \\
\hline
\end{tabular}




\section{Conclusions}

The survival of patients with STBI which were ventilated by consistent combination of forced ventilation with the pressure control (CPV) and 2 patterns of accessory lung venti- lation: Constant Positive Airway Pressure (CPAP) and Biphasic positive airway pressure (BiPAP) is significantly higher than in the case of forced ventilation with the volume control with Positive end-expiratory pressure.

\section{References}

1. Bullock R. Neurotrauma. Guidelines for the Management of Severe Traumatic Brain Injury 2007; 24: 101-106.

2. Churlyaev A. Acute respiratory distress syndrome with severe traumatic brain injury. Common resuscitation 2009; 2: 21-27.

3. Shekhovtseva K. Epidemiology of traumatic brain injury and the organization of assistance to victims of Stavropol Territory. Dissertation for the candidate of medical sciences: Russian Research Neurosurgical institute - St. Petersburg, 2006. www. dissercat.com.

4. Saidov S. Violation of hypo coagulating lung function in severe traumatic brain injury. Dissertation for the candidate of medical sciences: Moscow, 2004. www.dissercat.com.

5. Arabi Y, Haddad S, Tamim H. Mortality Reduction after Implementing a Clinical Practice Guidelines-Based Management Protocol for Severe Traumatic Brain Injury. J CritCare 2010; 25: 190-195.

6. Lewandowski K, Lewandowski M: Epidemiology of ARDS. Minerva Anestesiol 2006; 72: 473-477.

7. Eloise M Harman. Acute Respiratory Distress Syndrome Treatment \& Management. eMedicineHealth 2014, 2, www// emedicine.medscape.com.

8. Atul Malhotra. Benefits of Low Tidal Volume Ventilation in ARDS. Crit Care Clin 2008; 18: 79-89.

9. Luce J. Corticosteroids in ARDS. An evidencebased review. Crit Care Clin 2002; 1: 79-89.

10. Meduri G. Effect of prolonged methylprednisolone therapy in unresolving acute respiratory distress syndrome. A randomized controlled trial. JAMA 2008; 280: 159-165.
11. Meduri G. Methylprednisolone infusion in early severe ARDS: results of a randomized controlled trial. Chest 2007; 131: 954-963.

12. Steinberg K. Efficacy and safety of corticosteroids for persistent acute respiratory distress syndrome. N Engl J Med 2007; 354: 1671-1684.

13. Meduri G. Steroid treatment in ARDS: a critical appraisal of the ARDS network trial and the recent literature. Intensive Care Medicine 2008; 34: 61-69.

14. Izaguirre-Anariba D. Acute Respiratory Distress Syndrome: A Complex Clinical Condition, Medscape 2015. www. reference.medscape.com

15. Roberts I, Yates D, Sandercock P. CRASH trial collaborators. Effect of intravenous corticosteroids on death within 14 days in 10008 adults with clinically significant head injury (MRC CRASH trial): randomised placebo-controlled trial. Lancet 2008; 364: 1321-1328.

16. Martin-Loeches I, Lisboa T, Rhodes A. Use of early corticosteroid therapy on ICU admission in patients affected ey severe pandemic (H1N1) v influenza A infection. Intensive Care Med. Feb 2011; 37(2): 272-283.

17. MacLaren R, Jung R. Stress-dose corticosteroid therapy for sepsis and acute lung injury or acute respiratory distress syndrome in critically ill adults. Pharmacotherapy 2002; 22: 1140-1156.

18. Agarwal R, Aggarwal A Etiology and outcomes of pulmonary and extrapulmonary acute lung injury/ARDS in a respiratory ICU in North India. Chest 2006; 130: 724-729.

19. Brun-Buisson C, Richard J, Mercat A. Early Corticosteroids in Severe Influenza A/H1N1 Pneumonia and Acute Respiratory Distress Syndrome. Am J Respir Crit Care Med 2011; 183: 1200-1206.

Received: 2016-11-29 